\title{
High Redshift Radio Galaxies
}

\author{
James S. Dunlop \\ Astrophysics, Liverpool John Moores University, Byrom Street, Liverpool L3 $3 A F$, UK
}

\begin{abstract}
The potentially important role of jet-cloud interactions in determining the appearance of high-redshift radio galaxies is discussed and investigated via new 3-dimensional simulations of off-axis jet-cloud collisions. The results indicate that the most powerful radio sources are likely to be observed during or shortly after a jet-cloud interaction, and that such interactions can explain both the radio structures and the spatial association between optical and radio light found in powerful radio galaxies at high redshift. It is argued that, due to the radio-power dependence of such complicating effects, the optical-infrared colours and morphologies of very radio-luminous high-redshift galaxies can tell us essentially nothing about their evolutionary state. Either one must study much less radio-luminous sources in which the AGN-induced contamination is minimised, or one must attempt to determine what fraction of the baryonic mass of the radio galaxy has been converted into stars at the epoch of observation. Recent observations aimed at performing the latter experiment on two well-known high-redshift radio galaxies $(4 \mathrm{C} 41.17 \mathrm{\&}$ B2 $0902+34$ ) are described. It is concluded that at present there exists no clear evidence that either of these famous galaxies is 'primæval'; on the contrary, the continued low-dispersion of the infrared Hubble diagram at $z>2$ points toward a much higher redshift of formation for elliptical galaxies.
\end{abstract}

Key words: cosmology: observations, galaxies: active, galaxies: formation

\section{Introduction}

The evolved stellar populations and low gas masses of present-day elliptical galaxies imply that they formed most of their stars in a rapid burst at high redshift, but an unambiguous example of a 'primæval' elliptical has yet to be discovered. Since radio galaxies are identified exclusively with ellipticals at low redshift, their highredshift $(z>2)$ counterparts are obvious candidate primæval ellipticals. However, although recent studies have suggested that at least some high-redshift radio galaxies may be very young (Eales et al. 1993; Eales \& Rawlings 1993), uncertainities remain because of the mounting evidence that high radio luminosity couples to distorted ultraviolet and optical properties through processes associated with nuclear activity (Dunlop \& Peacock 1993). In this paper I consider one possible cause of this radio-luminosity dependent distortion, namely the interaction between the radio-source jet and dense gas clouds in the intra/inter-galaxy medium. I then discuss whether, given such complications, there exists any clear evidence that high-redshift radio galaxies really are primæval, focusing on new observations of two famous high-redshift galaxies and concluding with a re-appraisal of the radio-galaxy infrared Hubble diagram at $z>2$. 


\section{The interaction between the radio source and its environment}

\subsection{Observational eVIDEnCE}

Following the discovery that the optical and radio structures of many high-redshift radio galaxies are closely aligned (McCarthy et al. 1987; Chambers, Miley \& van Breugel 1987) it has become increasingly clear that the optical and radio properties of such objects are much more closely linked than had been previously supposed. In fact a considerable body of evidence already existed to suggest that interaction with inhomogeneities in the surrounding medium was important in determining the appearance of many high-redshift radio sources (e.g. Lonsdale \& Barthel 1986; Barthel 1987), but the alignment effect prompted consideration of the potential reciprocal ability of the radio jet to influence the surrounding medium (e.g. Rees 1989). Since then the discovery of a number of important correlations has led to the suggestion that the environment may be the dominant factor in determining the appearance of powerful radio galaxies (Dunlop \& Peacock 1993). Of particular note are i) the discovery by McCarthy, van Breugel \& Kapahi (1991) that the radio and optical line-emission asymmetries in high-redshift radio galaxies are correlated in the sense that the brighter line emission occurs on the side of the nearer radiolobe; ii) the discovery by Liu \& Pooley (1991) that the radio lobe with the steeper spectral index is virtually always the nearer and more depolarized lobe, and iii) the discovery that both the level of blue light and the strength of the alignment effect are correlated with a mix of radio power and spectral index (Dunlop \& Peacock 1993). The implication is that alignments arise because of a selection effect, with higher radio luminosities and steeper spectra arising when a radio jet encounters dense material (Eales 1992; Dunlop \& Peacock 1993).

\subsection{Numerical Simulation of Jet-Cloud interactions}

From theoretical considerations, a radio source of given beam power is certainly expected to produce a higher luminosity in a high-density environment (Williams 1985), and observationally the most luminous radio sources are known to reside in regions of high galaxy density (Yates, Miller \& Peacock 1989; Hill \& Lilly 1990).

The effect of off-axis jet-cloud collisions has already been investigated via 3dimensional numerical simulations by De Young (1991). However, these simulations used the 'beam scheme' (Sanders \& Prendergast 1974) which is only 1st-order accurate, and has proved unsuitable for some astrophysical problems (van Albada et al. 1982). In addition they did not involve proper monitoring of the separate progress of jet and ambient material; tracer particles were used to provide some indication of the behaviour of the jet but calculation of synchrotron emission from jet material was not possible.

At present, therefore, the detailed effects of jet-cloud interactions in extragalactic radio sources have not been simulated with sufficient sophistication to enable meaningful comparison with the growing body of observational evidence described above. In an attempt to fill this important gap, I, in collaboration with 
Tim O'Brien and Steve Higgins at Liverpool, have initiated a new programme of 3-dimensional simulations the first results of which are presented here.

The simulations are carried out with a numerical hydrodynamics code based on the second-order Godunov method of Falle (1991) which solves the inviscid Euler equations with an adiabatic equation of state in a 3-dimensional cartesian co-ordinate system. In addition to the usual dynamical variables, we have also computed a parameter representing the advection of jet material through the grid. This allows us to easily distinguish jet material from ambient material. We then assume that the magnetic field is frozen into the jet plasma, so that the field energy density $U_{m a g}$ is related directly to the density of the jet material. If, in addition, we assume that the energy density of particles accelerated to relativistic energies in shocks can be simply related to the thermal energy density in the gas, we can then calculate the intensity of the expected synchrotron emission in a straightforward manner. These simplifying assumptions enable us to produce synthetic radio maps directly from the hydrodynamic results (Higgins, O'Brien \& Dunlop 1995).

Example results from our initial simulations are shown in Figure 1. In this case the jet has a velocity of $0.1 \mathrm{c}$, a radius of $0.05 \mathrm{kpc}$, is in pressure balance with the ambient medium and is at the same density. The cloud is also in pressure balance, and has a density of 100 times the ambient density, and a radius of $0.7 \mathrm{kpc}$. Figure 1 gives synthetic radio map contour plots (after convolution with a gaussian representing the observing beam) and corresponding greyscale plots of density at four different epochs during the interaction of the jet with the gas cloud $(t=0.16$, $0.32,0.48$ and 0.64 million years).

The preliminary results from these simulations display several interesting features. First, this simple off-axis collision with a single cloud can produce a rather complex radio structure which clearly evolves through the simulation. There are three main features - a hotspot at the deflection point, a hotspot at the head of the jet and a secondary hotspot - but the relative brightness of these features changes rapidly with time. Second, as a consequence of the jet-cloud interaction, the radio source becomes much more luminous. Third, after collision the gas cloud becomes elongated and closely aligned to the radio structure.

\subsection{IMPLICATIONS FOR HIGH-REDSHIFT RADIO GALAXIES}

As expected, the radio source brightens dramatically from its pre-impact luminosity when the jet impacts upon the gas cloud. However, the simulations also indicate that increased radio luminosity persists for a considerable time after the initial collision, albeit at a gradually decreasing level. Given the relative time-scales involved it would thus seem reasonable to suggest that samples of ultra-luminous radio sources are likely to be dominated by sources which have 'recently' been involved in a jet-cloud interaction. Such a selection bias favouring sources which are 'just past their best' could be accentuated by the technique of ultra steep spectrum selection which has been so successful in locating luminous sources at high redshift (Rottgering 1992). 

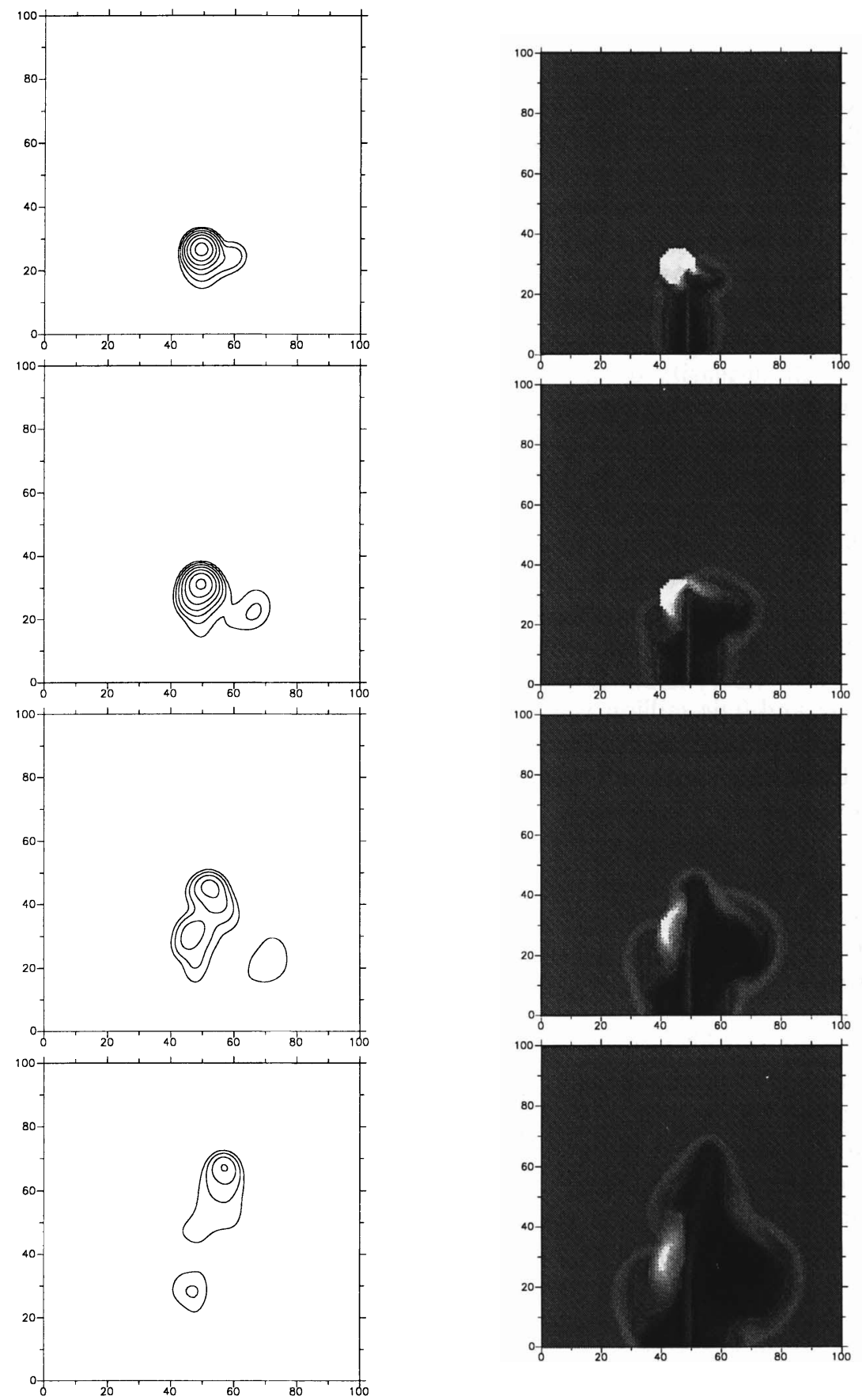

Fig. 1. Results from new 3-D simulations of a jet-cloud interaction. See text for details. 
These simulations also predict that the jets in powerful/steep-spectrum radio sources should be 'bordered' by the elongated remnants of the disrupted gas cloud. If we suppose that such gas cloud fragments are the sites of line emission and scattering of continuum from a hidden quasar, then it is to be expected that samples of such sources will display a strong alignment effect between their optical and radio structures. In addition, the simulations show that the apparent accuracy of the spatial correspondence between the optical and radio structures is expected to vary considerably depending upon the precise epoch of observation. This simple fact can explain some of the apparently different manifestations of the alignment effect observed in different sources. For example, a very close spatial correspondence between the radio and optical structures, such as is observed in the $z=3.8$ radio galaxy 4C41.17 (Miley et al. 1992) can be produced shortly after the initial collision; in Fig 1c the interaction is producing multiple hotspots such as are observed in 4C41.17 (Carilli, Owen \& Harris 1993), and these radio hotspots lie within $1 \mathrm{kpc}$ of the aligned optical light. At later times (e.g. Fig. 1d) the predicted spatial correspondence between the radio and optical structures is much less exact due to fact that the head of the radio jet has moved beyond the cloud, but nevertheless the optical and radio structures remain clearly aligned. Such a structure is observed in, for example, the $z=1.2$ radio galaxy 3C324 (Rigler et al. 1992; Dickinson 1995). By this stage in the collision the radio and optical axes appear to be misaligned by $\simeq 20^{\circ}$ due to the combined effects of jet diversion and cloud disruption. Such a apparent optical-radio misalignment is exactly what is observed in 3C324 and indeed is typical of that found in most 'aligned' high-redshift radio galaxies.

Several other observed aspects of the alignment effect can be explained by this type of jet-cloud interaction. First it leads naturally to the observed correlation between radio power/spectral index and the statistical prevalence of the alignment effect (Dunlop \& Peacock 1993). Second, unlike some other proposed explanations of the alignment effect (e.g. Daly 1992), these simulations predict that, even in well-aligned radio galaxies, the optical emission will in general not appear precisely co-spatial with the radio emission if viewed with sufficient resolution. Recent HST images of $3 \mathrm{C} 368$ have shown that this appears to be the case, with much of the optical emission being resolved into curved filaments which border the radio structure rather than being precisely coincident with it (van Breugel priv. comm.). Third, if the interacting gas cloud is in fact another galaxy, then the (undiverted) stars in this galaxy may contribute to the aligned light, providing one possible explanation of the radio-infrared alignments seen in many high-redshift radio galaxies (Dunlop \& Peacock 1993). 


\section{The ages of high-redshift radio galaxies}

\subsection{AGN-InDUCED CONTAMINATION}

Regardless of whether jet-cloud interactions of the type described in the previous section are the primary cause of optical-radio alignments, it is now clear that the optical/infrared colours and morphologies of powerful high-redshift radio galaxies are of little help in determining the true age of the dominant stellar population in the host galaxy. At optical/ultraviolet wavelengths emission from material around the jet (emission-lines, scattered quasar continuum and starlight, either from jetinduced starformation or from pre-existing stars in an interacting galaxy) will in general make the galaxy appear artificially blue as well as giving it a misleadingly complex morphology. Conversely, while it might be thought that near-infrared observations would yield a more undistorted view of the starlight, the presence of a dust-obscured quasar can make the galaxy appear excessively nucleated, red and luminous at near-infrared wavelengths (e.g. McCarthy, Persson \& West 1992; Lacy et al. 1995).

Given the above it is reasonable to ask what, if anything, can be done to determine the true age of high-redshift radio galaxies. One solution is to study the optical-infrared properties of much less radio-luminous sources in which the effect of the AGN should be insignificant (Dunlop \& Peacock 1995). A second, and complementary, approach is to attempt to constrain what fraction of the presentday baryonic mass of a giant elliptical galaxy has been processed into stars in high-redshift radio galaxies at the epoch of observation. In the remainder of this section I describe two recent (and very different) attempts to perform the latter experiment on two famous high-redshift galaxies, both of which have been hailed as primæval galaxies - B2 $0902+34$, for several years the most distant known galaxy ( $z=3.4$; Lilly 1988$)$, and $4 \mathrm{C} 41.17$, the current holder of this title $(z=3.8$; Chambers, Miley \& van Breugel 1990).

\subsection{Sub-millimetre Photometry of 4C41.17}

On the basis of its rather blue ultraviolet-optical spectral energy distribution and complex multi-component morphology, it has been claimed that $4 \mathrm{C} 41.17$ may be a genuine example of a primæval elliptical galaxy (Chambers, Miley \& van Breugel 1990; Miley et al. 1992). However, as stressed above, the contaminating effects of the AGN at ultraviolet-optical wavelengths are expected to be severe for such a luminous steep-spectrum radio source. In addition the discovery that the radio core of this galaxy (Carilli, Owen \& Harris 1993) is located at the position of a gap in the ultra-violet emission provides strong circumstantial evidence that its shortwavelength morphology is further complicated by significant dust obscuration. Consequently it seems that the best, and perhaps only way of determining whether this galaxy really is primæval is to determine the mass of gas which has yet to be converted into stars at the epoch of observation. We have recently attempted to do this via a determination of dust mass based on sub-millimetre photometry 
(Dunlop et al. 1994), and have succeeded in detecting 4C 41.17 at $800 \mu m$ using the JCMT (Figure 2). As discussed by Dunlop et al. (1994) this detection combined with an upper limit at $450 \mu \mathrm{m}$ leads to an estimate of the gas mass of $10^{11} M_{\odot}$, or $\sim 10 \%$ of the stellar mass of the most luminous elliptical galaxies. Taken at face value this result suggests that while $4 \mathrm{C} 41.17$ may well be involved in a spectacular interaction/starburst, it need not be genuinely primæval. In fact none of the currently available data on $4 \mathrm{C} 41.17$ can exclude the possibility that the bulk of its stellar population formed at much higher redshifts.

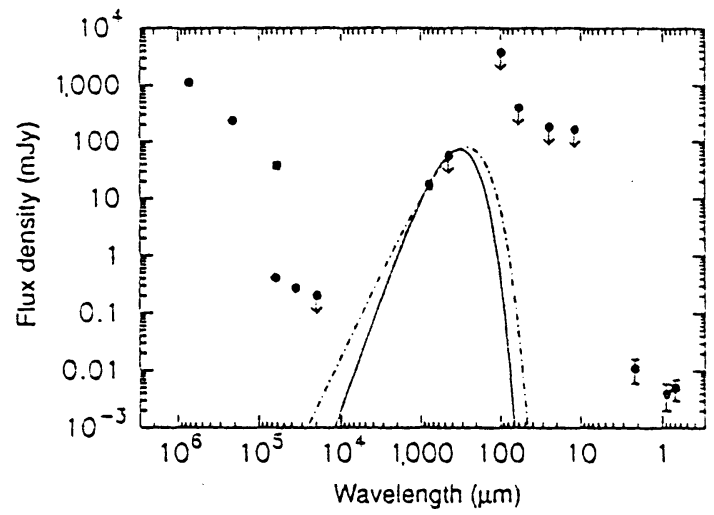

Fig. 2. The radio-optical spectrum of $4 \mathrm{C} 41.17$ showing the far-infrared excess due to dust emission as detected by sub-millimetre photometry.

\subsection{Deep K-BAnd Imaging of B2 0902+34}

At the time of its discovery, the apparently red optical-infrared colour of the radio galaxy B2 0902+34 generated great interest because it implied that the stellar population in this $z=3.4$ radio galaxy was already $\sim 1-2$ Gyr old (Lilly 1988). Since then this result has been turned on its head by the discovery that the true 4 -arcsec aperture magnitude of $0902+34$ is a magnitude fainter than measured by Lilly (1988), and that even this fainter $K$-band luminosity is in fact dominated by emission lines (Eales \& Rawlings 1993). 0902+34 has thus re-entered the limelight, but this time cited as perhaps the best candidate for a primæval galaxy on account of its line-corrected flat-spectrum spectral energy distribution, and its apparently low optical luminosity (Eales et al. 1993).

Nevertheless some confusion has remained because, independent of his 1988 infrared image, Lilly (1989) reported three single-element large-aperture ( $T .4$ $12.4 \mathrm{arcsec}$ ) photometry measurements of $0902+34$ between 1983 and 1987 , all of which yielded $K<19$. This led Eisenhardt \& Dickinson (1992) to attempt to investigate the $K$-band morphology of $0902+34$ but they concluded that this could not be reliably attempted until the advent of larger format arrays.

Figure 3 shows a new deep (162-minute) $K$-band image of $0902+34$ obtained 
using the new large-format $(256 \times 256)$ IRCAM3 camera at UKIRT in May 1994 . This image is, of course, contaminated by emission lines, but interestingly it yields an 8-arcsec software aperture magnitude of $K=18.7 \pm 0.05$, consistent with the original value determined by Lilly, and a 4-arcsec magnitude of $K=19.7 \pm 0.05$ consistent with the value measured by Eales et al. (1993). This shows that the apparent disagreement of a magnitude between the original aperture magnitude and the more recent 4-arcsec imager-determined magnitude is due to the fact that $0902+34$ is very extended at $K$. In fact, as illustrated in Fig. 3, the de Vaucouleurs scale-length $r_{e}$ appears to be $\simeq 100 \mathrm{kpc}$ (for $\Omega_{0}=1, H_{0}=50 \mathrm{~km} \mathrm{~s}^{-1}, \mathrm{Mpc}^{-1}$ ). If as concluded by Eisenhardt \& Dickinson (1992) the [OIII] emission only dominates within the central 3-4 arcsec, this would suggest that $0902+34$ may not in fact be under-luminous, merely very extended.
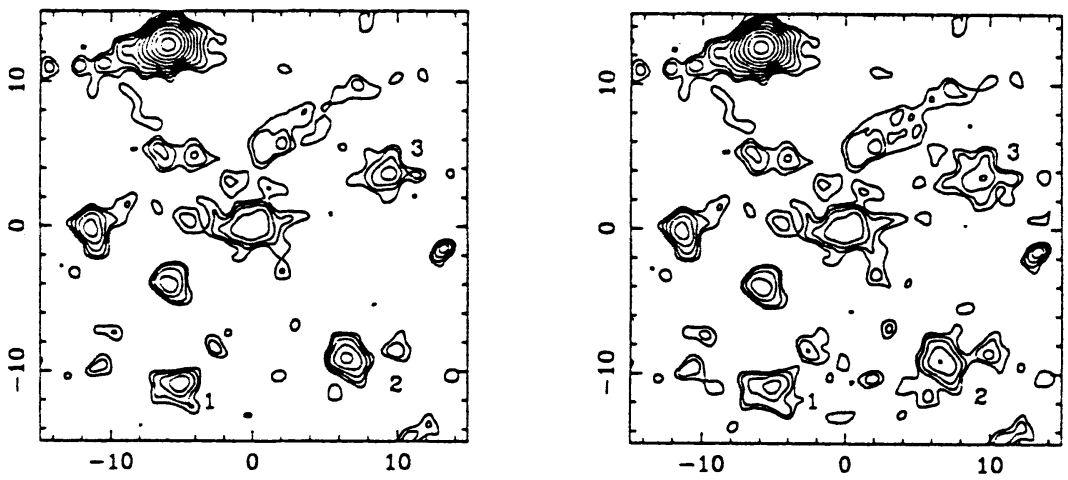

Fig. 3. A new deep $\mathrm{K}$-band image of $\mathrm{B} 20902+34$. The galaxies numbered 1,2 and 3 are synthesized galaxies of scale-length $30 \mathrm{kpc}$ in the $\mathrm{LH}$ plot and $100 \mathrm{kpc}$ in the RH plot.

\section{Conclusion - the $\mathrm{K}-\mathrm{z}$ diagram for radio galaxies}

If there exists no clear evidence that high-redshift radio galaxies are primæval, is there any counter-evidence that they are old? One such piece of evidence is provided by the continued low dispersion of the radio-galaxy $K-z$ diagram at high redshift. Although Eales \& Rawlings (1993) have demonstrated that the scatter in this relation appears to rise dramatically at $z>2$, if one adopts larger aperture $(8-\operatorname{arcsec})$ measurements at high-redshift this effect seems to disappear (Figure 4). The difference is undoubtedly in part due to the increased sensitivity of the smaller apertures ( 4 arcsec) used by Eales \& Rawlings to different quasar contributions in different sources. It may, however, also be telling us that significant dynamical evolution is occurring at $z \simeq 3$. It will be of interest to see whether comparably deep infrared imaging of other high-redshift radio galaxies shows them to be as unusually extended as $0902+34$. 


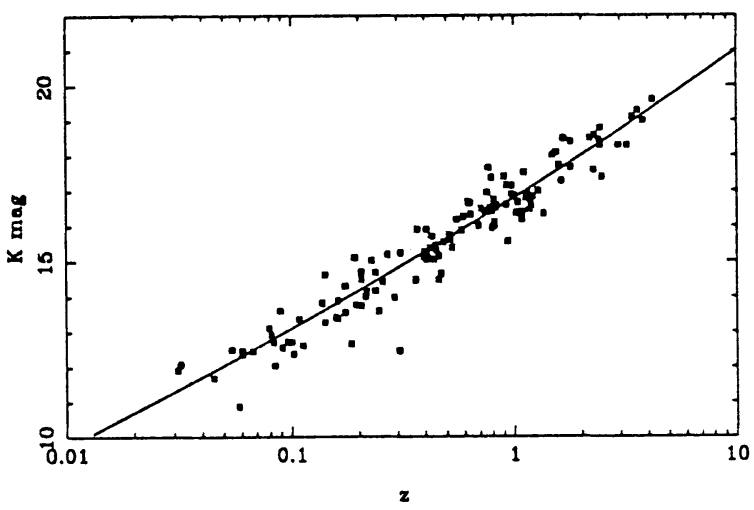

Fig. 4. The $\mathrm{K}-\mathrm{z}$ diagram for powerful radio galaxies using large aperture data at high $\mathrm{z}$.

\section{References}

Barthel, P.D. \& Miley, G.K., 1987. Nature, 333, 319.

Carilli, C.L., Owen, F. \& Harris, D.E., 1993. AJ, 107, 480.

Chambers, K.C., Miley, G.K. \& van Breugel, W.J.M., 1987. Nature, 329, 624.

Chambers, K.C., Miley, G.K. \& van Breugel, W.J.M., 1990. ApJ, 363, 21.

Daly, R.A., 1992. ApJ, 386, L9.

De Young, D.S., 1991. ApJ, 371, 69.

Dickinson, M., 1995. In: 'Galaxies in the Young Universe', Ringberg Workshop Sep 1994, in press.

Dunlop, J.S. \& Peacock, J.A., 1993. MNRAS, 263, 936.

Dunlop, J.S. \& Peacock, J.A., 1995. In: 'Galaxies in the Young Universe', Ringberg Workshop Sep 1994, in press.

Dunlop, J.S. et al., 1994. Nature, 370, 347.

Eales, S.A., 1992. ApJ, 397, 49.

Eales, S.A. \& Rawlings, S., 1993. ApJ, 411, 67.

Eales, S.A. et al. 1993. Nature, 363, 140.

Eisenhardt, P. \& Dickinson, M., 1992. ApJ, 399, L47.

Falle, S.A.E.G., 1991. MNRAS, 250, 581.

Higgins, S.W., O'Brien, T.J. \& Dunlop, J.S., 1995. MNRAS, in preparation.

Hill, G.J. \& Lilly, S.J., 1990. ApJ, 367, 1.

Lacy, M. et al., 1995. MNRAS, in press.

Lilly, S.J., 1988. ApJ, 333, 161.

Lilly, S.J., 1989. $A p J, 340,77$.

Liu, R. \& Pooley, G., 1991. MNRAS, 249, 343.

Lonsdale, C.J. \& Barthel, P.D., 1986. ApJ, 303, 617.

McCarthy, P.J. et al. 1987. ApJ, 321, L29.

McCarthy, P.J., van Breugel, W. \& Kapahi, V.K., 1991. ApJ, 371, 478.

McCarthy, P.J., Persson, S.E. \& West, S.C., 1992. ApJ, 386, 52.

Miley, G.K. et al. 1992. ApJ, 401, L69.

Rees, M.J., 1989. MNRAS, 239, 1 P.

Rigler, M.A. et al. 1992. ApJ, 385, 61.

Rottgering, H., 1993. PhD Thesis, University of Leiden.

Sanders, R.H. \& Prendergast, K.H., 1974. ApJ, 188, 489.

van Albada, G.D., van Leer, B. \& Roberts, W.W., 1982. A\&A, 108, 76.

Williams, A.G., 1985. PhD Thesis, University of Cambridge.

Yates, M.G., Miller, L. \& Peacock, J.A., 1989. MNRAS, 240, 129. 